\title{
Changes in the gene expression levels of microRNAs in the rat hippocampus by sevoflurane and propofol anesthesia
}

\author{
GENTARO GOTO, YOKO HORI, MASASHI ISHIKAWA, SHUNSUKE TANAKA and ATSUHIRO SAKAMOTO
}

\author{
Department of Anesthesiology and Pain Medicine, Graduate School of Medicine, \\ Nippon Medical School, Tokyo 113-8603, Japan
}

Received September 24, 2013; Accepted February 13, 2014

DOI: $10.3892 / \mathrm{mmr} .2014 .2038$

\begin{abstract}
General anesthesia is commonly used in the surgical arena, but little is known regarding its influence at the genomic and molecular levels. MicroRNAs (miRNAs) belong to a new class of non-coding RNA molecules which influence cell biology. In the present study, it was hypothesized that miRNAs alter gene expression levels under general anesthesia. The aim was to compare the miRNA expression profiles in the rat hippocampus in response to anesthesia with representative volatile (sevoflurane) and intravenous (propofol) anesthetics. Wistar Rats were randomly assigned to either a $2.4 \%$ sevoflurane, $600 \mu \mathrm{g} / \mathrm{kg} / \mathrm{min}$ propofol or control (without anesthetics) group. Total RNA from hippocampal samples which contained miRNA was subjected to quantitative reverse transcriptionpolymerase chain reaction and Taqman Low-Density Arrays (TLDA). A total of 373 miRNAs are associated with rats and the TLDA analysis revealed that 279 expressed miRNAs (74.8\%) were expressed in all three groups. Significant differences in the levels of 33 of the 279 expressed miRNAs (11.8\%) were observed among the three groups in response to the anesthetic agents, and when compared with the control group, significant differences were found in 26 of the 279 expressed miRNAs $(9.3 \%)$. Following sevoflurane anesthesia, the levels of four miRNAs were significantly increased and those of 12 were significantly reduced. By contrast, following propofol anesthesia, the levels of 11 miRNAs were significantly reduced but no miRNAs exhibited significantly elevated levels. Fourteen miRNAs were significantly differentially expressed between the two anesthesia groups. In conclusion, sevoflurane and propofol exerted different effects on miRNA expression in the rat hippocampus.
\end{abstract}

Correspondence to: Dr Gentaro Goto, Department of Anesthesiology and Pain Medicine, Graduate School of Medicine, Nippon Medical School, 1-1-5 Sendagi, Bunkyo-ku, Tokyo 113-8603, Japan

E-mail: gntrgt@nms.ac.jp

Key words: microRNA, sevoflurane, propofol, hippocampus, rat

\section{Introduction}

General anesthetics act on the central nervous system, mainly the brain and the spinal cord, to induce general anesthesia. General anesthesia has commonly been used in the surgical arena for $\sim 150$ years, and its efficacy and safety have been assured by clinical outcomes (1-3). However, little is known regarding its comprehensive influence at the genomic and molecular levels, which is not reflected by mortality and morbidity. Sevoflurane is an inhalational anesthetic frequently used in Japan (4). It has low solubility in blood and tissues, thus there is usually a rapid recovery from anesthesia. Propofol is the most commonly used intravenous anesthetic and is administered as an alkylphenol formulated in a lipid emulsion $(5,6)$. Propofol provides rapid and smooth induction of anesthesia and exhibits rapid clearance from the body.

Due to rapid progress in the field of genomics, microarray technology allows the analysis of genome-wide changes in gene expression in cells and tissues. Several studies regarding general anesthetics, including volatile and intravenous anesthetics, have been performed and they have found that general anesthetics affect gene expression in the rat central nervous system. To elucidate cellular function, exhaustive analyses of the expression of genes (genomics), proteins (proteomics) and metabolites (metabolomics) have been performed in the brain of rats, which is the main target of general anesthetics. In a previous genomic study, the inhalation anesthetic sevoflurane affected the expression of $1.5 \%$ of genes in various rat organs, as detected using a microarray analysis (7). General anesthesia altered the expression levels of genes involved in circadian rhythms; persistent suppression of several circadian genes was identified following treatment of rats with the inhalation anesthetic sevoflurane (8) and the intravenous anesthetics propofol and dexmedetomidine (9). In a proteomics study, the protein expression in the rat brain was regulated differentially according to the anesthetic agent used: The inhalation anesthetic sevoflurane or the intravenous anesthetic propofol (10). In a metabolomics study, the inhalation anesthetic isoflurane and the intravenous anesthetic propofol exerted differential effects on the brain metabolism of rats (11). Based on these previous studies, it was hypothesized that gene expression, protein expression and metabolism are different in the rat brain under inhalation anesthesia and intravenous anesthesia. 
A

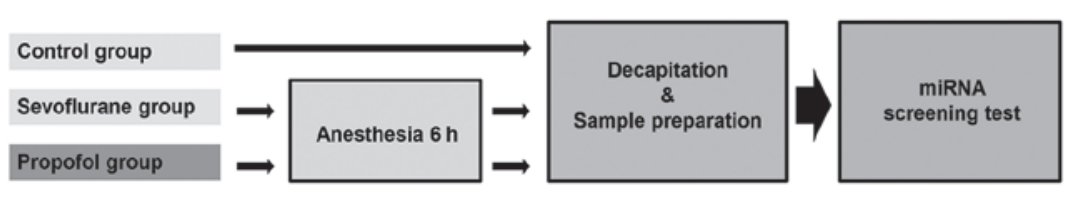

B

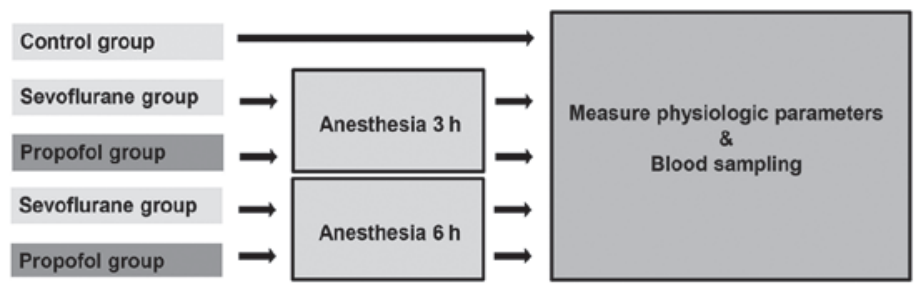

Figure 1. Flow chart of the experimental protocols. (A) Experimental protocol of miRNA screening test. (B) Experimental protocol for measurement of physiological parameters. miRNA, microRNA.

MicroRNAs (miRNAs) are a class of highly conserved, short, single-stranded, non-coding RNA molecules. miRNAs are 20-24 nucleotides in length and are encoded by the genome. miRNAs bind to imperfectly complementary sequences in the 3'-untranslated region (3'-UTR) of target messenger RNAs (mRNAs) and regulate gene expression at the post-transcriptional level; that is, inhibiting mRNA translation and/or inducing mRNA degeneration (12). A study has shown that miRNAs negatively regulate $>30 \%$ of mammalian gene expression (13). Furthermore, single miRNAs may bind with hundreds of unique mRNAs, thus altering the expression of multiple cellular pathways (14). miRNAs are important in the central nervous system as they affect various post-transcriptional regulation mechanisms, including cytoskeletal/neuronal growth, cellular metabolism, biosynthesis, stress/death responses, signal transduction and synaptic plasticity (15-18). There is evidence that miRNAs have specific temporal and spatial expression patterns in the mammalian brain (19). However, the gene regulatory networks of the majority of miRNAs in the brain have not yet been fully elucidated.

General anesthetics were developed empirically in clinical settings. This type of anesthetic is essential in producing analgesia, immobility, unconsciousness and memory blockade (amnesia). As certain patients experience unexpected recall of events during surgery, intraoperative amnesia has been a desired endpoint of general anesthesia from the perspectives of the patient and the anesthesiologist. Understanding the mechanisms underlying amnesia is a matter of particular importance. The hippocampus is considered to be an important organ in cognitive function and episodic memory processes and, as reported by Pan et al (20), administration of the inhalation anesthetic sevoflurane resulted in altered gene expression levels of mRNAs that affect memory function in the rat hippocampus.

To the best of our knowledge, no published studies have addressed the association between sevoflurane anesthesia and the expression of miRNAs in the rat hippocampus. It was hypothesized that the miRNAs that influence mRNA expression also alter gene expression levels in the hippocampus under sevoflurane anesthesia. In addition, the inhalation anesthetic sevoflurane and the intravenous anesthetic propofol were predicted to induce different effects on the expression levels of miRNAs in the rat brain. Thus, the aim of the present study was to compare the miRNA expression profiles in the rat hippocampus in response to anesthesia with representative volatile (sevoflurane) and intravenous (propofol) anesthetics. To the best of our knowledge, this is the first attempt to perform an exhaustive profiling analysis of miRNA expression in the hippocampus under general anesthesia.

\section{Materials and methods}

Experimental protocol. A previous study model (21-23) on miRNA data acquisition, processing and statistical analysis was used. A flow chart of the experimental protocol is shown in Fig. 1.

Animals. Animal protocols, including the method for euthanasia, were approved by the Animal Experimental Ethics Review Committee of Nippon Medical School (Tokyo, Japan; approval no.: 24-005). All procedures followed the guidelines of the International Association for the Study of Pain (24). Specific pathogen-free male Wistar rats aged six weeks old, (Saitama Experimental Animal Supply Co., Saitama, Japan) were purchased and acclimated under temperature-controlled conditions on a 12-h light/dark cycle, starting at 06:00 and 18:00, respectively, for a week prior to the commencement of experiments. All rats received food and water ad libitum, and the average starting weight of the rats was $250 \pm 10 \mathrm{~g}$.

\section{Procedures for administration of anesthetic and termination.} During the experiment, each rat was housed in a plastic box $(45 \times 32 \times 23 \mathrm{~cm})$, which was large enough for the rat to move and breathe fresh air. The rats were minimally handled in order to reduce stress. The tails of the rats were placed through a small hole in the wall of the box, a catheter was inserted into the tail vein without anesthesia and normal saline was infused continuously at a rate of $1.0 \mathrm{ml} / \mathrm{h}$. The rats were allowed to breathe spontaneously and were supplied with an air-oxygen mixture [fraction of inspired oxygen $\left(\mathrm{FiO}_{2}\right)=0.40$ ]. Body temperature was maintained at $37.0^{\circ} \mathrm{C}$ with a heat lamp.

The rats were randomized into three groups $(n=7$ in each group) and each group received either sevoflurane, propofol 
Table I. Physiological data for the control, sevoflurane and propofol anesthesia groups.

\begin{tabular}{|c|c|c|c|c|c|}
\hline & \multirow{2}{*}{$\frac{\text { Control }}{3 \mathrm{~h}}$} & \multicolumn{2}{|c|}{ Sevoflurane } & \multicolumn{2}{|c|}{ Propofol } \\
\hline & & $3 \mathrm{~h}$ & $6 \mathrm{~h}$ & $3 \mathrm{~h}$ & $6 \mathrm{~h}$ \\
\hline $\mathrm{pH}$ & $7.44 \pm 0.02$ & $7.43 \pm 0.03$ & $7.42 \pm 0.02$ & $7.41 \pm 0.02$ & $7.41 \pm 0.03$ \\
\hline $\mathrm{PaO}_{2}(\mathrm{mmHg})$ & $137.5 \pm 10.5$ & $135.6 \pm 12.5$ & $131.2 \pm 14.5$ & $129.3 \pm 10.6$ & $130.5 \pm 8.6$ \\
\hline $\mathrm{PaCO}_{2}(\mathrm{mmHg})$ & $36.5 \pm 2.3$ & $39.8 \pm 2.9$ & $40.5 \pm 3.3$ & $41.5 \pm 3.2$ & $41.7 \pm 2.5$ \\
\hline Heart rate (beats/min) & $331 \pm 17$ & $324 \pm 19$ & $331 \pm 21$ & $315 \pm 12$ & $320 \pm 22$ \\
\hline Mean arterial pressure $(\mathrm{mmHg})$ & $120 \pm 9$ & $111 \pm 12$ & $117 \pm 13$ & $109 \pm 15$ & $112 \pm 13$ \\
\hline Body temperature $\left({ }^{\circ} \mathrm{C}\right)$ & $37.1 \pm 0.2$ & $37.0 \pm 0.1$ & $37.0 \pm 0.1$ & $36.9 \pm 0.2$ & $37.0 \pm 0.2$ \\
\hline
\end{tabular}

Analyses of variance followed by Tukey-Kramer test were used. No between-group differences were identified as significant. Data are presented as the mean \pm standard deviation of seven animals in each group.

or no anesthetic (control group). In the anesthesia groups, the rats were anesthetized with sevoflurane or propofol for $6 \mathrm{~h}$ (between 9:00 and 15:00). Rats undergoing inhalational anesthesia (sevoflurane) were placed in plastic boxes supplied with sevoflurane (Sevofrane ${ }^{\circledR}$; Maruishi Pharmaceutical Co., Ltd., Osaka, Japan; 2.4\% gas-air mixture) (25) and 40\% oxygen (6 1/min). Normal saline was administered via a venous catheter at a rate of $1.0 \mathrm{ml} / \mathrm{h}$. Rats undergoing intravenous anesthesia (propofol) were housed in plastic boxes supplied with $40 \%$ oxygen at a rate of $6 \mathrm{l} / \mathrm{min}$ and were administered $1 \%$ propofol (Diprivan ${ }^{\circledR}$; AstraZeneca, Osaka, Japan; $600 \mu \mathrm{g} / \mathrm{kg} / \mathrm{min}$ ) (26) via a venous catheter (rate of $9.0 \mathrm{ml} / \mathrm{h}$ for rats weighing $250 \mathrm{~g}$ ). In the control group, the rats were not administered anesthetics; however, they were similarly housed in plastic boxes supplied with $40 \%$ oxygen at a rate of $6 \mathrm{l} / \mathrm{min}$ and normal saline was administered via a venous catheter at a rate of $1.0 \mathrm{ml} / \mathrm{h}$. Rats in the control group were not allowed access to food and water. All rats were allowed to breathe spontaneously. Animals were sacrificed immediately following completion of anesthesia at 15:00. Within 3 mins after decapitation, the whole brain was rapidly removed and the bilateral hippocampus was directly removed from the brain, as described previously $(27,28)$.

Physiological parameters. Physiological variables were measured in three separate groups of rats not used for hippocampal miRNA evaluation ( $n=7$ each), which were treated with sevoflurane, propofol or no anesthetic (control group). For all rats, a catheter was inserted into the tail vein without anesthesia and normal saline was infused continuously at a rate of $1.0 \mathrm{ml} / \mathrm{h}$. During the experiment, each rat was placed in a plastic cage $(45 \times 32 \times 23 \mathrm{~cm})$ supplied with an air-oxygen mixture $\left[\left(\mathrm{FiO}_{2}\right)=0.40\right]$ and allowed to breathe spontaneously. The body temperatures of the rats were maintained at $37.0^{\circ} \mathrm{C}$ with a heat lamp and their rectal temperatures were measured. As a surgical procedure, the left femoral artery was cannulated to obtain blood samples for measuring the arterial $\mathrm{PO}_{2}$, arterial $\mathrm{PCO}_{2}$, arterial blood $\mathrm{pH}$, heart rate and arterial blood pressure (Table I). Anesthetics were administered as described for the miRNA analysis groups. In the sevoflurane and propofol physiological groups, anesthesia was maintained for an additional 3 or $6 \mathrm{~h}$ after surgery. In the control group, the rats were anesthetized by intraperitoneal injection of sodium pentobarbital $(50 \mathrm{mg} / \mathrm{kg})$ for the surgery and it was maintained for $3 \mathrm{~h}$. The rats were placed in a rat tunnel in the plastic cages and allowed to recover from the anesthesia. Blood samples were obtained from all five groups.

RNA extraction and miRNA screening test; Taqman Low-Density Arrays (TLDA). Hippocampal samples were washed twice with cold phosphate-buffered saline, immediately placed in RNAlater solution (Applied Biosystems, Inc., Foster City, CA, USA) and stored at $-80^{\circ} \mathrm{C}$ for one day. Following storage, the samples were defrosted and the RNAlater solution was rapidly separated from the samples by centrifugation $\left(10,000 \times \mathrm{g}, 4^{\circ} \mathrm{C}, 5 \mathrm{~min}\right)$. Total RNA was extracted from each sample using a mirVana ${ }^{\mathrm{TM}}$ miRNA Isolation kit (Applied Biosystems, Inc.) according to the manufacturer's instructions. RNA quantity and quality were assessed by measuring absorbance using a NanoDrop ND-1000 (Thermo Fisher Scientific, Waltham, MA, USA). Any RNA samples with a 260/280 nm absorbance ratio $>1.8$ were subjected to quantitative analysis. Total RNA samples containing miRNA were used for quantitative reverse transcription-polymerase chain reaction (qRT-PCR).

The miRNA expression profiles in the hippocampal samples were analyzed using TLDA Rodent miRNA cards v.3.0 A and B (Applied Biosystems, Inc.), a set of miRNA low-density arrays which enables simultaneous qRT-PCR for 373 preloaded rat miRNA targets and three endogenous controls (Mamm U6, U87 and Y1). All miRNA targets are cataloged in the miRBase database (http://www.mirbase.org/). All procedures were based on those of a previous study (29). Briefly, TLDA was performed as a two-step process. During the first step, 1,200 ng of total RNA per sample was reverse-transcribed using Megaplex RT Primer Pools A and B (Applied Biosystems, Inc.), up to 381 stem-looped primers per pool and a TaqMan miRNA Reverse Transcription kit (Applied Biosystems, Inc.). In the second step, each of the resulting complementary DNA pools was diluted, mixed with TaqMan Universal PCR Master mix (Applied Biosystems, Inc.) and deionized distilled water (Wako Pure Chemical Industries, Ltd., Tokyo, Japan), and loaded into one of the eight fill ports on the TLDA microfluidic card. The cards were 
Table II. Differential expression of miRNAs between the sevoflurane anesthesia and control groups using TLDA.

\begin{tabular}{lcc}
\hline Assay & Sevoflurane (FC) & Raw P-value \\
\hline hsa-miR-423-3P & $1.87 \pm 0.62$ & 0.021 \\
mmu-miR-24-2\# & $1.42 \pm 0.37$ & 0.032 \\
rno-miR-664 & $1.50 \pm 0.50$ & 0.033 \\
hsa-miR-9\# & $1.25 \pm 0.24$ & 0.048 \\
mmu-miR-103 & $0.71 \pm 0.15$ & 0.007 \\
mmu-miR-125b-5p & $0.71 \pm 0.15$ & 0.010 \\
mmu-miR-142-3p & $0.63 \pm 0.14$ & 0.010 \\
mmu-miR-101b & $0.72 \pm 0.48$ & 0.010 \\
mmu-miR-27b & $0.77 \pm 0.14$ & 0.019 \\
mmu-miR-499 & $0.37 \pm 0.12$ & 0.019 \\
mmu-miR-30b & $0.64 \pm 0.18$ & 0.029 \\
mmu-miR-19b & $0.70 \pm 0.23$ & 0.030 \\
rno-miR-351 & $0.42 \pm 0.17$ & 0.038 \\
mmu-miR-129-3p & $0.74 \pm 0.18$ & 0.040 \\
mmu-miR-369-5p & $0.70 \pm 0.22$ & 0.040 \\
rno-miR-219-2-3p & $0.70 \pm 0.22$ & 0.049 \\
\hline
\end{tabular}

Expression levels of the miRNAs were significantly increased or reduced in the sevoflurane anesthesia group compared with those in the control group, as determined by TLDA. Values are the mean fold change \pm standard deviation. TLDA, TaqMan low-density array; FC, fold change; miRNA, microRNA; hsa, Homo sapiens; mmu, Mus musculus; rno, Rattus norvegicus. Raw P-value, statistical results of Tukey-Kramer test.

briefly centrifuged for $1 \mathrm{~min}$ at $315 \mathrm{x} \mathrm{g}$ to distribute samples to the multiple wells connected to the fill ports and were then sealed to prevent well-to-well contamination. The cards were processed and analyzed using a 7900HT Fast Real-Time PCR system (Applied Biosystems, Inc.).

Data analysis was performed using DataAssist software v2.0 (Applied Biosystems, Inc.). The resulting data were expressed as threshold cycle $(\mathrm{Ct})$ values, where $\mathrm{Ct}$ represents a unitless value defined as the fractional cycle number at which the sample fluorescence signal passes a fixed threshold above baseline. The relative quantities of all the miRNAs were calculated by the comparative $\mathrm{Ct}$ method. $\Delta \mathrm{Ct}$ is the difference in $\mathrm{Ct}$ values derived from the experimental samples and the controls, and $\Delta \Delta \mathrm{Ct}$ represents the difference between paired samples, as calculated by the following formula: $\Delta \Delta \mathrm{Ct}=\Delta \mathrm{Ct}$ of sample following anesthesia - $\Delta \mathrm{Ct}$ of the control group. The expression ratio shows the relative quantity of the target gene (Xtarget) to the control gene (Xcontrol). The fold change was computed by the following formula: Xtarget/Xcontrol $=2^{-\Delta \Delta \mathrm{Ct}}$. The $2^{-\Delta \Delta \mathrm{Ct}}$ method is a convenient method for analyzing the relative changes in gene expression from qRT-PCR experiments. Relative quantification (RQ) values (relative levels of miRNA expression) were calculated by this comparative $\mathrm{Ct}$ method using RQ Manager version 1.2.1 (Applied Biosystems, Inc.) (30). Graphic displays were visualized as heat map results of hierarchical clustering. Distances between samples and assays were calculated for hierarchical clustering as determined by $\Delta \mathrm{Ct}$ values, using Euclidean distance.
Table III. Differential expression of miRNAs between the propofol anesthesia and control groups using TLDA.

\begin{tabular}{lcc}
\hline Assay & Propofol (FC) & Raw P-value \\
\hline rno-miR-20b & $0.56 \pm 0.30$ & 0.011 \\
rno-miR-125b\# & $0.60 \pm 0.37$ & 0.012 \\
mmu-miR-221 & $0.63 \pm 0.18$ & 0.021 \\
mmu-miR-99a & $0.37 \pm 0.64$ & 0.023 \\
mmu-miR-142-3p & $0.67 \pm 0.14$ & 0.024 \\
mmu-miR-181a & $0.60 \pm 0.32$ & 0.024 \\
mmu-miR-324-5p & $0.69 \pm 0.23$ & 0.033 \\
mmu-miR-451 & $0.42 \pm 0.15$ & 0.034 \\
hsa-miR-99b\# & $0.17 \pm 0.15$ & 0.035 \\
mmu-miR-376b & $0.67 \pm 0.22$ & 0.041 \\
rno-miR-207 & $0.41 \pm 0.15$ & 0.049 \\
\hline
\end{tabular}

Expression levels of the miRNAs were significantly decreased in the propofol anesthesia group compared with those in the control group, as determined by TLDA. Values are the mean fold change \pm standard deviation. TLDA, TaqMan low-density array; FC, fold change; miRNA, microRNA; hsa, Homo sapiens; mmu, Mus musculus; rno, Rattus norvegicus. Raw P-value, statistical results of Tukey Kramer test.

Statistical analysis. Values are expressed as the mean \pm standard deviation. Analysis of variance (ANOVA) followed by Tukey-Kramer test was used to compare the physiological data and miRNA expression levels in the sevoflurane anesthesia, propofol anesthesia and control groups. $\mathrm{P}<0.05$ was considered to indicate a statistically significant difference in all tests. Tukey-Kramer test was applied to identify genes and miRNAs that were significantly differentially expressed on exposure to anesthetic agents. ANOVA followed by Tukey-Kramer test was performed using Kyplot software, version 5.0 (KyensLab Incorporated, Tokyo, Japan).

\section{Results}

Anesthesia of rats. Following induction of sevoflurane or propofol anesthesia, all rats lost consciousness within $5 \mathrm{~min}$, with loss of corneal reflexes and the withdrawal response to pain. No rats moved spontaneously or died until the end of anesthesia. Data for all animals were used.

Physiological data. The possible effects of anesthesia on a number of physiological parameters were investigated in three separate groups of rats. Fig. 1 shows a flow chart of the experimental protocol. The physiological data for the treatment groups during anesthesia were compared with those of the control group, in which no anesthesia was administered following the termination of anesthesia for the cannulation of the femoral artery. Low mean blood pressure and high $\mathrm{PCO}_{2}$ were observed following the administration of anesthetic drugs. However, no significant differences in the physiological parameters among the administration groups and control group were identified (Table I). Hypoxia, hyper/hypocapnia, hypotension or hypothermia were not observed in any of the three groups. 
Table IV. Differential expression of miRNAs between the sevoflurane and propofol anesthesia groups using TLDA.

\begin{tabular}{lccc}
\hline Assay & Sevoflurane (FC) & Propofol (FC) & Raw P-value \\
\hline hsa-miR-9\# & $1.25 \pm 0.24$ & $0.88 \pm 0.11$ & 0.002 \\
rno-miR-125b\# & $1.14 \pm 0.12$ & $0.60 \pm 0.37$ & 0.002 \\
hsa-miR-324-3p & $1.38 \pm 0.57$ & $0.75 \pm 0.08$ & 0.004 \\
mmu-miR-674\# & $1.27 \pm 0.27$ & $0.78 \pm 0.21$ & 0.006 \\
mmu-miR-101b & $1.49 \pm 0.47$ & $0.98 \pm 0.12$ & 0.007 \\
hsa-miR-423-3P & $1.87 \pm 0.62$ & $1.05 \pm 0.59$ & 0.011 \\
hsa-miR-140-3p & $1.60 \pm 0.80$ & $0.82 \pm 0.17$ & 0.015 \\
hsa-miR-206 & $1.76 \pm 1.07$ & $0.70 \pm 0.45$ & 0.019 \\
mmu-miR-24-2\# & $1.42 \pm 0.37$ & $0.99 \pm 0.24$ & 0.028 \\
rno-miR-664 & $1.50 \pm 0.50$ & $0.99 \pm 0.21$ & 0.033 \\
rno-miR-409-3P & $1.52 \pm 0.59$ & $0.91 \pm 0.22$ & 0.037 \\
mmu-miR-770-5p & $1.64 \pm 0.95$ & $0.84 \pm 0.25$ & 0.041 \\
mmu-miR-29c & $0.99 \pm 0.27$ & $0.61 \pm 0.44$ & 0.042 \\
mmu-miR-374-5p & $1.26 \pm 0.51$ & 0.042 \\
\hline
\end{tabular}

Expression levels of the miRNAs were significantly differentially expressed between the sevoflurane and the propofol anesthesia groups, as determined by TLDA. Values are the mean fold change \pm standard deviation in each group. TLDA, TaqMan low-density array; FC, fold change; miRNA, microRNA; hsa, Homo sapiens; mmu, Mus musculus; rno, Rattus norvegicus. Raw P-value, statistical results of Tukey-Kramer test.

miRNA screening test: TLDA. In order to identify suitable endogenous controls, three different candidate miRNAs were analyzed for variance in gene expression with DataAssist software v2.0. The statistical method ranked the candidate endogenous control genes with an excellent correlation of raw stability values. The most stably expressed Y1 was selected as the endogenous control and the relative miRNA expression levels were normalized against $\mathrm{Y} 1$. $\mathrm{Ct}$ values $>35$ indicated that their expression levels were too low for accurate analysis. The miRNA expression $\mathrm{Ct}$ value was therefore standardized at $\leq 35$.

The results of the TLDA analysis of the hippocampi are shown in Tables II-IV ( $\mathrm{n}=7$ for each group; significance level, $\mathrm{P}<0.05)$. There were 750 miRNAs on the TLDA plate; 373 miRNAs were associated with rats and the rest with mice. TLDA analysis revealed 279 expressed miRNAs (74.8\%), which were expressed in the sevoflurane, propofol and control groups. Significant differences were observed in the levels of 33 of the 279 expressed miRNAs (11.8\%) among the three groups in response to the anesthetic agents, and when compared with the control group, significant differences were found in 26 of the 279 expressed miRNAs (9.3\%). Following sevoflurane anesthesia, the levels of four miRNAs were significantly increased and those of 12 were significantly reduced compared with those of the control group. By contrast, following propofol anesthesia, the levels of 11 miRNAs were significantly reduced but no miRNAs exhibited significantly elevated levels. Of the total 33 significantly differentially expressed miRNAs, one miRNA was common to both anesthetics, whereas 14 miRNAs were significantly differentially expressed (Tables II-IV).

Cluster analysis from TLDA data is shown in Fig. 2. Significantly differentially expressed miRNAs were converted into a heat map in order to improve the understanding of the
miRNA expression patterns in each group. Heat maps for the calculated clusters are commonly used gene-expression analyses and are constructed for visualization of gene expression levels. In the present study, heat maps illustrated the gene expression levels from the hippocampal samples following treatment with anesthetics using $\Delta \mathrm{CT}$ values. The color intensity on the heat map corresponds to the absolute intensity of the miRNA expression. Each column represents a sample and each row shows miRNA in the heat map. By individually clustering columns and rows, the heat map simultaneously presents the separate samples and miRNA clustering in one graphic. The dendrograms of clustering analysis for the samples and miRNAs are shown to the top and left of the heat map. This indicated the relatedness of the samples as determined by the overall miRNA expression values. The dendrogram separates into three main branches: The sevoflurane anesthesia, the propofol anesthesia and the control groups.

\section{Discussion}

The present study demonstrated that sevoflurane and propofol anesthesia induced numerous changes in the miRNA expression levels of the rat hippocampus. Using TLDA data, the hierarchical clustering with heat map presented the semantic similarities and expression patterns of miRNA. Hierarchical clustering separated the samples into three distinct branches in the dendrogram: The sevoflurane anesthesia group, the propofol anesthesia group and the control group (no anesthetic). These findings indicate that the miRNA expression patterns with general anesthetic treatment were distinct from those of the control group. In addition, the inhalation anesthetic sevoflurane and the intravenous anesthetic propofol exerted different effects on the miRNA expression in the rat hippocampus. 


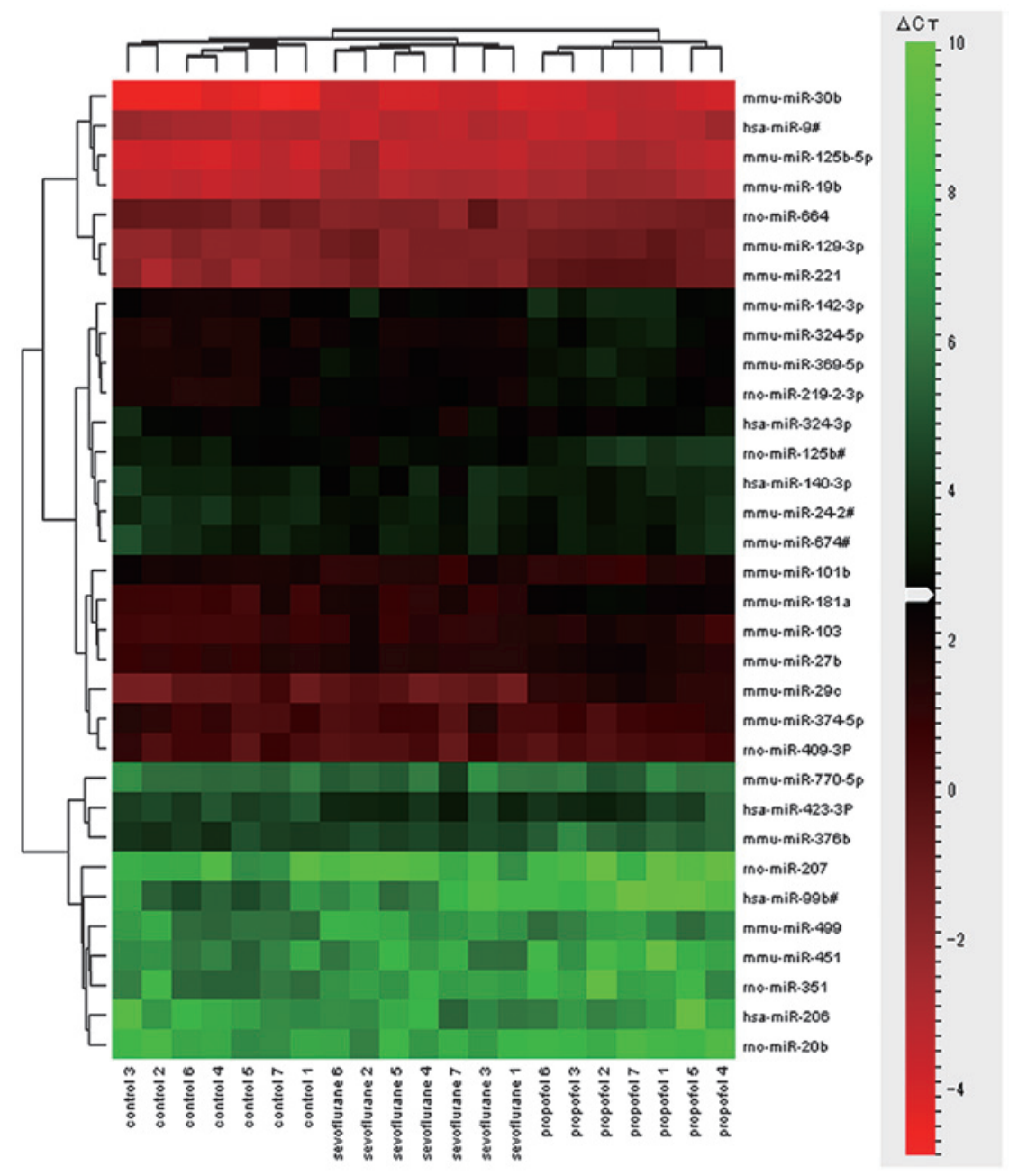

Figure 2. Unsupervised hierarchical cluster analysis. There were 33 miRNAs differentially expressed in response to sevoflurane and propofol anesthesia, as determined by their relative expression levels. miRNA profiles of control and anesthetized rats were visualized with agglomerative hierarchical clustering using Euclidean distance from TaqMan Low-Density arrays. Columns correspond to samples and are labeled to indicate whether a column represents a sample from the sevoflurane anesthesia group, the propofol anesthesia group or the control group. Each row corresponds to an individual miRNA sequence. The miRNA names and the dendrogram for miRNA clustering are displayed on the left. The dendrogram of the sample clustering revealed that samples were separated into three groups: The control group, the sevoflurane group and the propofol group. Colors indicate the miRNA expression variance: Red indicates higher gene expression levels, green indicates lower expression levels and black indicates the median value. miRNA, microRNA; mmu, Mus musculus; hsa, Homo sapiens; rno, Rattus norvegicus.

In the present study, experiments were conducted using the inhaled anesthetic sevoflurane and the intravenous anesthetic propofol. Sevoflurane was selected as it is one of the most commonly used inhaled clinical anesthetic agents in Japan (4). Propofol was selected as it is a widely used intravenous anesthetic in everyday clinical practice in a number of countries. The two drugs have been used extensively in previous studies concerning the effects of anesthetic agents on gene expression (7-9), protein expression (10) and metabolites (11) in the rat brain. Changes in the miRNA expression levels in the hippocampi of rats anesthetized with one minimum alveolar concentration of inhaled anesthetics (sevoflurane at $2.4 \%$; 22) or with continuous infusion of intravenous anesthetics (propofol at $600 \mu \mathrm{g} / \mathrm{kg} / \mathrm{min} ; 23$ ) were investigated in the present study. Infusion of propofol at $600 \mu \mathrm{g} / \mathrm{kg} / \mathrm{min}$ is the median effective dose for rats (26). The two doses were selected since lower doses may not induce adequate anesthesia and higher doses may produce hemodynamic changes $(10,22)$ that may independently alter hippocampal miRNA expression levels.
A number of miRNAs determined to be differentially expressed in the present study are known to be involved in stem cell self-renewal, synaptic plasticity and memory consolidation in the rat hippocampus. For example, miR-125b, a neuronal miRNA, is important in proliferation and differentiation of neural stem cells (31). miRNA-181a is rich in mature nerve cells and bioinformatic analysis has revealed that the cAMP response element-binding protein 1 (CREB1) mRNA 3'-UTR contains a complementary sequence to the miR-181a seed region. CREB1 is a key nuclear factor highly expressed in hippocampal neurons on which numerous signaling pathways converge (32-34). In the present study, the expression levels of these miRNAs were found to be significantly affected by sevoflurane and/or propofol anesthesia; therefore, general anesthesia may affect these cell signaling pathways, including synaptic plasticity and memory consolidation. In addition, the changes in the levels of miRNA may be attenuated following general anesthesia.

It was hypothesized that sevoflurane and propofol anesthesia induce different expression patterns of miRNA 
in the rat hippocampus. Sevoflurane and propofol induce general anesthesia but have different mechanisms of anesthetic action. Single miRNAs may bind with hundreds of unique mRNAs, thus regulating the expression of multiple cellular pathways, and individual miRNAs reciprocally act on mRNA. The TLDA data of the present study were not satisfactory to determine the validity of this hypothesis since they provide only a snapshot of the changes in miRNA expression patterns. Pan et al (20) showed that sevoflurane anesthesia induces changes in the mRNA expression levels in the rat hippocampus, which may be associated with memory impairment or other neural disorders. Although changes in the levels of mRNA and miRNA expression are likely to affect subsequent protein expression, there is a lack of correlation between them. The final functional entities in cells are not mRNAs or miRNAs, but proteins that undergo numerous post-translational modifications, thereby controlling cellular mechanisms (35). Investigation of the action mechanisms of general anesthetics is a noteworthy field and further studies using molecular approaches are required for the elucidation of these mechanisms.

Due to advances in high-throughput technologies and bioinformatics, there are several methods for gene expression profiling. The results of microarray analyses are commonly validated using qRT-PCR, which is the most sensitive and reproducible method for quantifying gene expression. Single qRT-PCR assays and TLDA analyses rely on identical amplification technologies. Single qRT-PCR is a reliable method for the analysis of miRNA expression as the reactions are run in triplicate; by contrast, TLDA analyses are run individually. Hui et al (36) demonstrated that the technical reproducibility of TLDAs was high (intrasample correlations $>0.9$, accuracy 92.8\%). Furthermore, Mees et al (37) demonstrated that TLDA and single qRT-PCR correlated well with regards to the quantity and quality of the measured miRNAs. The TLDA analysis of the present study has the same procedures as those of previous studies $(21,22,38)$. In these studies, the single qRT-PCR results of individual miRNA were equivalent to the TLDA analysis results. These results confirmed that the TLDA analysis data of the present study were as accurate as those of single qRT-PCR and that the TLDA data were reliable.

The present study has certain limitations. The study was conducted in rats and innate differences in gene expression in different species of animals mean that extrapolation of results from rats to other species is difficult. Additionally, miRNA microarray analyses were conducted using whole hippocampal samples. The hippocampus is composed of the dentate gyrus and the cornu ammonis. The cornu ammonis is separated into three predominant divisions: CA1, CA 2 and CA3, but the results of the present study only reflected the whole hippocampal miRNA expression pattern. For further analyses, localization of gene-expression changes is required. Furthermore, a large number of miRNAs were measured; therefore, it is possible that certain results were false positives.

In conclusion, the data from the present study indicated changes in the miRNA expression levels in the rat hippocampus under general anesthesia. When compared with that of the control group, the inhalation anesthetic sevoflurane and the intravenous anesthetic propofol exerted differential effects on miRNA expression. In subsequent studies, the time-dependent changes in the levels of miRNA expression under general anesthesia may be further investigated, along with the post-anesthetic influence of miRNA at various time points.

\section{Acknowledgements}

This study was supported by a Grant-in-Aid for Science Research (C) (project no. 20591820) from the Japan Society for the Promotion of Science (Tokyo, Japan).

\section{References}

1. Forrest JB, Rehder K, Goldsmith $\mathrm{CH}$, et al: Multicenter study of general anesthesia. I. Design and patient demography. Anesthesiology 72: 252-261, 1990.

2. Brown BR Jr and Frink EJ: The safety of sevoflurane in humans. Anesthesiology 79: 201-203, 1993.

3. Marik PE: Propofol: therapeutic indications and side-effects. Curr Pharm Des 10: 3639-3649, 2004

4. Stachnik J: Inhaled anesthetic agents. Am J Health Syst Pharm 63: 623-634, 2006.

5. Eger EI 2nd: Characteristics of anesthetic agents used for induction and maintenance of general anesthesia. Am J Health Syst Pharm 61 (Suppl 4): S3-S10, 2004.

6. Simons PJ, Cockshott ID, Douglas EJ, Gordon EA, Hopkins K and Rowland M: Disposition in male volunteers of a subanaesthetic intravenous dose of an oil in water emulsion of 14C-propofol. Xenobiotica 18: 429-440, 1988.

7. Sakamoto A, Imai J, Nishikawa A, et al: Influence of inhalation anesthesia assessed by comprehensive gene expression profiling. Gene 356: 39-48, 2005.

8. Kobayashi K, Takemori K and Sakamoto A: Circadian gene expression is suppressed during sevoflurane anesthesia and the suppression persists after awakening. Brain Res 1185: 1-7, 2007.

9. Yoshida Y, Nakazato K, Takemori K, Kobayashi K and Sakamoto A: The influences of propofol and dexmedetomidine on circadian gene expression in rat brain. Brain Res Bull 79: 441-444, 2009.

10. Tsuboko Y and Sakamoto A: Propofol anaesthesia alters the cerebral proteome differently from sevoflurane anaesthesia. Biomed Res 32: 55-65, 2011.

11. Kawaguchi H, Hirakawa K, Miyauchi K, Koike K, Ohno Y and Sakamoto A: Pattern recognition analysis of proton nuclear magnetic resonance spectra of brain tissue extracts from rats anesthetized with propofol or isoflurane. PLoS One 5: e11172, 2010.

12. Bak M, Silahtaroglu A, Møller M, et al: MicroRNA expression in the adult mouse central nervous system. RNA 14: 432-444, 2008.

13. Miranda KC, Huynh T, Tay Y, et al: A pattern-based method for the identification of MicroRNA binding sites and their corresponding heteroduplexes. Cell 126: 1203-1217, 2006.

14. Brennecke J, Stark A, Russell RB and Cohen SM: Principles of microRNA-target recognition. PLoS Biol 3: e85, 2005.

15. Wayman GA, Davare M, Ando H, et al: An activity-regulated microRNA controls dendritic plasticity by down-regulating p250GAP. Proc Natl Acad Sci USA 105: 9093-9098, 2008.

16. Visvanathan J, Lee S, Lee B, Lee JW and Lee SK: The microRNA miR-124 antagonizes the anti-neural REST/SCP1 pathway during embryonic CNS development. Genes Dev 21: 744-749, 2007.

17. Bredy TW, Lin Q, Wei W, Baker-Andresen D and Mattick JS: MicroRNA regulation of neural plasticity and memory. Neurobiol Learn Mem 96: 89-94, 2011.

18. Lee $\mathrm{K}, \mathrm{Kim} \mathrm{JH}$, Kwon OB, et al: An activity-regulated microRNA, miR-188, controls dendritic plasticity and synaptic transmission by downregulating neuropilin-2. J Neurosci 32: 5678-5687, 2012.

19. Kosik KS: The neuronal microRNA system. Nat Rev Neurosci 7: 911-920, 2006

20. Pan Z, Lu XF, Shao C, et al: The effects of sevoflurane anesthesia on rat hippocampus: a genomic expression analysis. Brain Res 1381: 124-133, 2011.

21. Ishikawa M, Tanaka S, Arai M, Genda Y and Sakamoto A: differences in microRNA changes of healthy rat liver between sevoflurane and propofol anesthesia. Anesthesiology 117: $1245-1252,2012$ 
22. Tanaka S, Ishikawa M, Arai M, Genda Y and Sakamoto A: Changes in microRNA expression in rat lungs caused by sevoflurane anesthesia: a TaqMan ${ }^{\circledR}$ low-density array study. Biomed Res 33: 255-263, 2012.

23. Genda Y, Arai M, Ishikawa $M$, Tanaka $S$, Okabe $T$ and Sakamoto A: microRNA changes in the dorsal horn of the spinal cord of rats with chronic constriction injury: A TaqMan ${ }^{\circledR}$ Low Density Array study. Int J Mol Med 31: 129-137, 2013.

24. Ethical standards for investigations of experimental pain in animals. The Committee for Research and Ethical Issues of the International Association for the Study of Pain. Pain 9: 141-143, 1980.

25. Obal D, Preckel B, Scharbatke H, et al: One MAC of sevoflurane provides protection against reperfusion injury in the rat heart in vivo. Br J Anaesth 87: 905-911, 2001.

26. Orth M, Barter L, Dominguez C, Atherley R, Carstens E and Antognini JF: Halothane and propofol differentially affect electroencephalographic responses to noxious stimulation. Br J Anaesth 95: 477-484, 2005.

27. Chiu K, Lau WM,Lau HT, So KF and Chang RC: Micro-dissection of rat brain for RNA or protein extraction from specific brain region. J Vis Exp 7: 269, 2007.

28. Glowinski J and Iversen LL: Regional studies of catecholamines in the rat brain. I. The disposition of $[3 \mathrm{H}]$ norepinephrine, $[3 \mathrm{H}]$ dopamine and $[3 \mathrm{H}]$ dopa in various regions of the brain. J Neurochem 13: 655-669, 1966.

29. Wang B, Howel P, Bruheim S, et al: Systematic evaluation of three microRNA profiling platforms: microarray, beads array, and quantitative real-time PCR array. PLoS One 6: e17167, 2011.
30. Livak KJ and Schmittgen TD: Analysis of relative gene expression data using real-time quantitative PCR and the 2(-Delta Delta C(T)) Method. Methods 25: 402-408, 2001.

31. Skalnikova H, Vodicka P, Gadher SJ and Kovarova H: Proteomics of neural stem cells. Expert Rev Proteomics 5: 175-186, 2008.

32. Cui Y, Xiao Z, Han J, et al: MiR-125b orchestrates cell proliferation, differentiation and migration in neural stem/progenitor cells by targeting Nestin. BMC Neurosci 13: 116, 2012.

33. Saba R, Störchel PH, Aksoy-Aksel A, et al: Dopamine-regulated microRNA MiR-181a controls GluA2 surface expression in hippocampal neurons. Mol Cell Biol 32: 619-632, 2012.

34. Liu Y,Zhao Z, Yang F, Gao Y, Song J and Wan Y: microRNA-181a is involved in insulin-like growth factor-1-mediated regulation of the transcription factor CREB1. J Neurochem 126: 771-780, 2013.

35. Mozzachiodi R and Byrne JH: More than synaptic plasticity: role of nonsynaptic plasticity in learning and memory. Trends Neurosci 33: 17-26, 2010

36. Hui AB, Shi W, Boutros PC, et al: Robust global micro-RNA profiling with formalin-fixed paraffin-embedded breast cancer tissues. Lab Invest 89: 597-606, 2009.

37. Mees ST, Schleicher C, Mardin WA, Senninger N, Colombo-Benkmann $\mathrm{M}$ and Haier J: Analyzing miRNAs in ductal adenocarcinomas of the pancreas. J Surg Res 169: 241-246, 2011.

38. Arai M, Genda Y, Ishikawa M, Shunsuke T, Okabe T and Sakamoto A: The miRNA and mRNA changes in rat hippocampi after chronic constriction injury. Pain Med 14: 720-729, 2013. 